\title{
Nueva mutación identificada en el gen FOXP3 en lactante menor con diarrea crónica como manifestación de enteropatía autoinmune - Síndrome IPEX
}

\author{
New mutation in FOXP3 gene identified in an infant with chronic diarrhea \\ as manifestation of autoinmune enteropathy - IPEX syndrome
}

\author{
Clara Plata García ${ }^{\mathrm{a}, \mathrm{b}}$, Lorena Martín-Marín ${ }^{\mathrm{a}, \mathrm{b}}$, Angela Soler-Ramírez ${ }^{\mathrm{a}, \mathrm{b}}$, Jorge A. Rojas ${ }^{\mathrm{c}}$, María P. Salazar
}

aFacultad de Medicina, Pontificia Universidad Javeriana, Bogotá, Colombia

bervicio de Pediatria, Hospital Universitario San Ignacio, Bogotá, Colombia

¿Unidad de Genética, Hospital Universitario San Ignacio, Bogotá, Colombia

Recibido: 3 de octubre de 2019; Aceptado: 29 de enero de 2020

\section{¿Qué se sabe del tema que trata este estudio?}

Síndrome IPEX es una enfermedad genética por mutaciones del gen FOXP3, que produce desregulación inmune y compromete varios órganos y sistemas. No tiene un tratamiento específico y su mortalidad es alta.
¿Qué aporta este estudio a lo ya conocido?

Se describe una nueva mutación del gen FOXP3, en un paciente con expresión severa de la enfermedad.

\section{Resumen}

Introducción: El síndrome IPEX (inmunodesregulación, poliendocrinopatía y enteropatía autoinmune ligada a X) causado por mutaciones en el gen FOXP3, se caracteriza por diarrea prolongada, alteraciones endocrinológicas y dermatitis. El tratamiento consiste en la administración de medicamentos inmunosupresores, siendo el trasplante de médula ósea la única cura potencial. Objetivo: Describir una nueva mutación del gen FOXP3, así como los hallazgos y evolución de un paciente con síndrome IPEX. Caso Clínico: Lactante menor masculino que debutó al mes de vida con diarrea crónica, falla intestinal e infecciones recurrentes. Exámenes de laboratorio y biopsia intestinal sugerentes de enteropatía autoinmune. Durante el seguimiento, el paciente presentó refractariedad al manejo inmunosupresor con esteroides, ciclosporina y tacrolimus, falleciendo a los 7 meses de edad por complicaciones vasculares. Antecedente familiar por línea materna de múltiples muertes en hombres menores de 1 año. Ante la sospecha de síndrome IPEX se realizó exoma en trío que reportó una mutación probablemente patogénica en el gen FOXP3. Conclusión: Se documentó una nueva mutación del gen FOXP3 en paciente con síndrome IPEX. A pesar de la baja prevalencia de esta enfermedad, es importante el reconocimiento de síntomas no específicos pero sugerentes del diagnóstico.
Palabras clave:

Síndrome IPEX;

FOXP3;

Enteropatía

Autoinmune;

Diarrea 


\begin{abstract}
Introduction: The IPEX (immune dysregulation, polyendocrinopathy, enteropathy, X-linked) syndrome is caused by the mutations of the FOXP3 gene, characterized by persistent diarrhea, endocrine disorders, and dermatitis. The treatment is the administration of immunosuppressive drugs, where hematopoietic stem cell transplantation is the only potential cure. Objective: To describe a new FOXP3 gene mutation, as well as the findings and evolution of a patient with IPEX syndrome. Clinical Case: Male infant presenting at one month of age with chronic diarrhea, intestinal failure, and recurrent infections. Lab tests and intestinal biopsy suggested autoimmune enteropathy. During follow-up, the patient presented resistance to immunosuppressive treatment with corticosteroids, cyclosporine, and tacrolimus, dying at 7 months of age due to vascular complications. He had a maternal family history of multiple deaths of men under 1 year of age. IPEX syndrome was suspected therefore a trio whole-exome sequencing was performed that showed a probably pathogenic FOXP3 gene mutation. Conclusion: A new FOXP3 gene mutation is reported in a patient with IPEX syndrome. Despite the low prevalence of this disease, it is important to recognize non-specific but suggestive symptoms for its diagnosis.
\end{abstract}

\section{Keywords:}

IPEX Syndrome;

FOXP3; Autoimmune

Enteropathy; Diarrhea

\section{Introducción}

El síndrome IPEX (inmunodesregulación, poliendocrinopatía y enteropatía autoinmune ligada a X) tiene patrón de herencia recesivo ligado al cromosoma $\mathrm{X}$, siendo los varones casi exclusivamente los afectados. Las mujeres portadoras de variante patogénica son asintomáticas, los hombres afectados heredan la variante a todas sus hijas, que serán portadoras asintomáticas y a ninguno de sus hijos.

Esta patología es causada por mutaciones en el gen FOXP3 (FORKHEAD BOX P3), localizado en Xp11.23. Este gen codifica un factor de transcripción (proteina FOXP3 o escurfina) que consta de 431 aminoácidos. La proteína codificada se encuentra expresada principalmente en tejido linfoide y en células CD4+ - CD25+, y su función es regular la transcripción de otros genes involucrados en el desarrollo y la función de las células $\mathrm{T}$ reguladoras, encargadas de mediar la respuesta inmune y la tolerancia a autoantígenos ${ }^{1,2}$.

Las principales manifestaciones de este síndrome consisten en la triada de diarrea crónica severa por enteropatía autoinmune, alteraciones endocrinológicas y dermatológicas secundarias a una desregulación del sistema inmune. Pueden coexistir anemia hemolítica, trombocitopenia y glomerulonefritis ${ }^{3}$. El objetivo principal del manejo es la recuperación nutricional y la administración de medicamentos inmunosupresores para el control de la enfermedad. El trasplante de médula ósea es considerado hasta la fecha como la única terapia potencialmente curativa de la entidad.

El pronóstico es pobre, con una alta tasa de morbimortalidad entre el primer y el segundo año de vida. Se ha descrito refractariedad a la terapia inmunosupresora y efectos adversos como hepatotoxicidad, nefrotoxicidad y aumento de las infecciones ${ }^{4}$. De esta manera los pacientes requieren un abordaje multidisciplinario para su manejo.

El síndrome IPEX es una enfermedad hereditaria ultra huérfana (OMIM 304790) $)^{5}$, la literatura consultada reporta menos de 300 casos diagnosticados en el mundo. Debido a su baja prevalencia, el diagnóstico constituye un reto para el personal médico.

El objetivo principal de este artículo es describir una nueva mutación del gen FOXP3, así como los hallazgos y evolución de un paciente con síndrome IPEX.

\section{Caso Clínico}

Paciente masculino, producto del segundo embarazo de padres no consanguíneos, sin antecedentes perinatales de importancia e historia familiar con múltiples varones lactantes fallecidos por línea materna, incluido un hermano mayor sin causa clara de muerte y sin autopsia (figura 1). Nació a las 38 semanas de gestación, con peso de $2.840 \mathrm{~g}$ y longitud de $50 \mathrm{~cm}$, ambos en rango normal. Al mes de vida inició con diarrea crónica de alto gasto y deterioro progresivo de su estado nutricional con retraso de crecimiento, infecciones respiratorias, de vías urinarias y bacteremias asociadas a catéter.

Hospitalizado desde el inicio de los síntomas en otra institución, donde sospecharon alergia a la proteína de leche de vaca, recibiendo manejo con fórmula hidrolizada, aminoácidos libres y libre de carbohidratos, sin respuesta clínica, iniciándose soporte nutricional con nutrición parenteral total. Se realizaron exámenes de laboratorio: Calprotectina $(489,9 \mathrm{ug} / \mathrm{g}$, valor inespecífico en menores de 1 año), test de azúcares reductores en materia fecal negativo, elastasa pancreática baja $(15 \mathrm{mcg} / \mathrm{g}$, valor posiblemente alterado por diarrea de alto gasto), iontoforesis por pilocarpina normal 


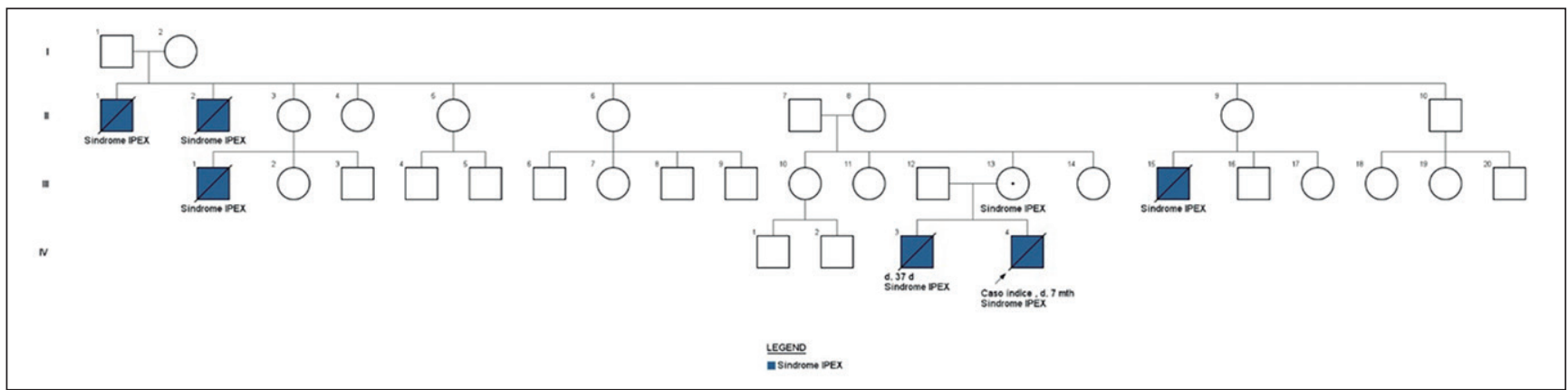

Figura 1. Árbol genealógico del caso indice en quien se realizó diagnóstico molecular, identificandose en él y su madre mutación a nivel del gen FOXP3.

Figura 2. Endoscopia de vías digestivas alta y baja: 1. Esófago; 2. Bulbo duodenal; 3. Segunda porción del duodeno; 4. Colón. Endoscopia alta con esófago de aspecto normal, bulbo duodenal y segunda porción con mucosa congestiva y aplanamiento de las vellosidades. Colon con eritema y friabilidad difusa de la mucosa y presencia de erosiones múltiples menores de $5 \mathrm{~mm}$ de diámetro.

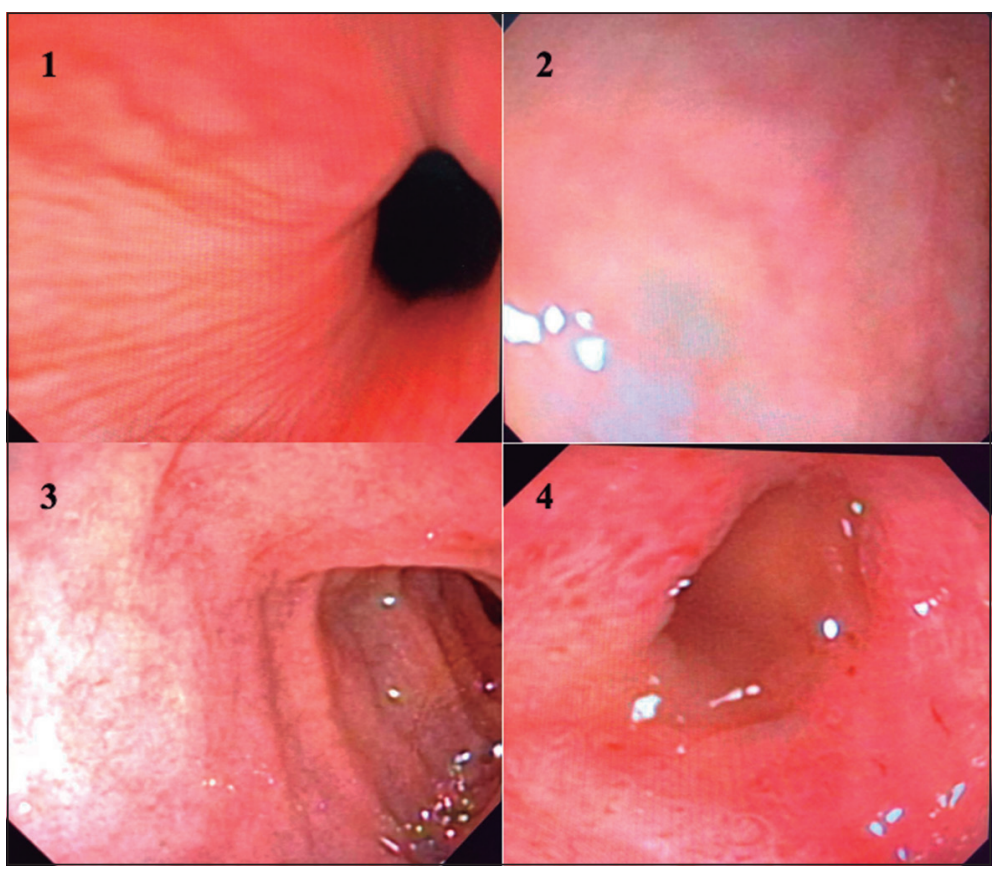

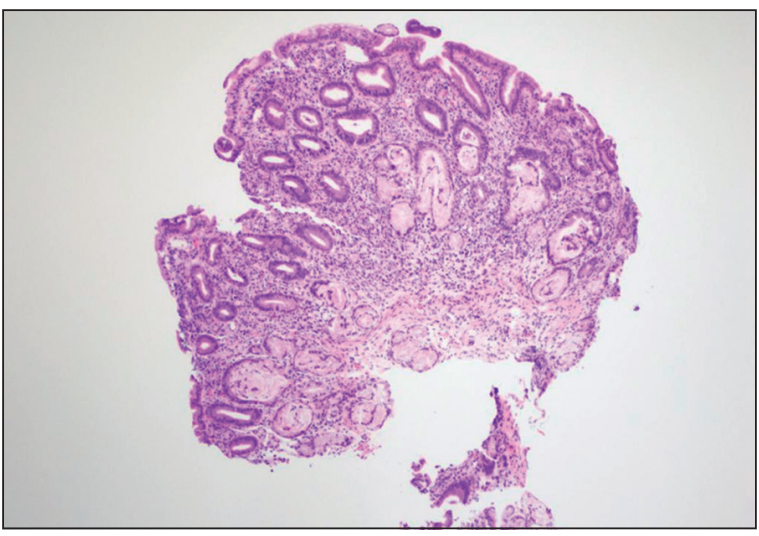

Figura 3. Biopsia duodenal con aplanamiento completo de vellosidades, ausencia de células caliciformes y de células de Paneth, y metaplasia pseudopilórica, con ensanchamiento de la lámina propia por infiltrado linfoplasmocitario y de neutrófilos. Cortesía de la Dra. Rocio del Pilar Lopez Panqueva.
(22 $\mathrm{mmol} / \mathrm{L}$ ) y perfil inmunológico con elevación de inmunoglobulina $\mathrm{E}(7.524 \mathrm{mg} / \mathrm{dl})$.

A los 3 meses y 15 días de vida fue derivado al Hospital Universitario San Ignacio de Bogotá para manejo por gastroenterología pediátrica, con peso de $2.340 \mathrm{~g}$ y longitud de $54 \mathrm{~cm}$ (Peso/longitud, longitud/edad y peso/edad $<-3 \mathrm{DE}, \mathrm{OMS})$.

Se realizó estudio endoscópico que mostró aplanamiento marcado de la mucosa duodenal y en colon mucosa congestiva e hiperémica (figura 2); la microscopia de luz en las biopsias identificó atrofia severa de vellosidades, ausencia de células de Paneth y de células caliciformes, lámina propia con aumento del infiltrado linfocitario con células apoptóticas, hallazgos sugestivos de enteropatía autoinmune (figura 3). Se toman exámenes complementarios y se descartaron otras patologías (tabla 1$)^{6}$. 
Tabla 1. Laboratorios

\begin{tabular}{|c|c|c|}
\hline Parámetro & Resultado & $\begin{array}{c}\text { Niveles de referencia } \\
\quad(3 \text { meses })\end{array}$ \\
\hline Inmunoglobulina A & $49,41 \mathrm{mg} / \mathrm{dl}$ & $4,6-46 \mathrm{mg} / \mathrm{dl}$ \\
\hline Inmunoglobulina G & $664,68 \mathrm{mg} / \mathrm{dl}$ & $176-581 \mathrm{mg} / \mathrm{dl}$ \\
\hline Inmunoglobulina M & $41,3 \mathrm{mg} / \mathrm{dl}$ & $24-89 \mathrm{mg} / \mathrm{dl}$ \\
\hline Inmunoglobulina E & $4131 \mathrm{mg} / \mathrm{dl}$ & $0,18-3,76 \mathrm{mg} / \mathrm{dl}$ \\
\hline CD3 & $63,2 \%$ & $51-77 \%$ \\
\hline CD4 & $51,9 \%$ & $35-56 \%$ \\
\hline CD8 & $11,7 \%$ & $12-23 \%$ \\
\hline CD19 & $12,88 \%$ & $11-41 \%$ \\
\hline Relación CD4/CD8 & $4,4 \%$ & \\
\hline Albumina & $1,8 \mathrm{~g} / \mathrm{dl}$ & $2,2-4,8 \mathrm{~g} / \mathrm{dl}$ \\
\hline TSH (Hormona estimulante tiroidea) & $0,99 \mathrm{mU} / \mathrm{l}$ & $0,58-5,57 \mathrm{mU} / \mathrm{l}$ \\
\hline Tiroxina libre & $0,94 \mathrm{ng} / \mathrm{dl}$ & $1,04-2,86 \mathrm{ng} / \mathrm{dl}$ \\
\hline Anticuerpos antiperoxidasa tiroidea & $18,4 \mathrm{IU} / \mathrm{ml}$ & \\
\hline Anticuerpos antitiroglobulina & $4,11 \mathrm{IU} / \mathrm{ml}$ & \\
\hline Coombs directo cualitativo & Negativo & \\
\hline Ferritina & $3.737 \mathrm{ng} / \mathrm{ml}$ & $50-200 \mathrm{ng} / \mathrm{ml}$ \\
\hline Vitamina B12 & $3.539 \mathrm{pg} / \mathrm{ml}$ & $200-835 \mathrm{pg} / \mathrm{ml}$ \\
\hline Anticuerpos antinucleares & Negativos & \\
\hline ENAS (Anticuerpos nucleares extractables) & Negativos & \\
\hline ANCAS (Anticuerpos anti citoplasma de neutrófilos) & Negativos & \\
\hline C4 (Complemento sérico componente 4) & $21,20 \mathrm{mg} / \mathrm{dl}$ & $8,7-27 \mathrm{mg} / \mathrm{dl}$ \\
\hline C3 (Complemento sérico componente 3) & $88 \mathrm{mg} / \mathrm{dl}$ & $64-131 \mathrm{mg} / \mathrm{dl}$ \\
\hline
\end{tabular}

Fuente: Hughes H, Kahl L. Manual Harriet Lane de pediatría. 21.a ed. Elsevier; 2018. 1244p.

Por antecedente familiar de múltiples lactantes masculinos fallecidos por línea materna, junto con el cuadro de diarrea crónica, las múltiples infecciones y el hallazgo de IgE elevada $(4.131 \mathrm{mg} / \mathrm{dl})$, se sospechó enteropatía autoinmune secundaria a inmunodeficiencia primaria ligada a X. Se solicitó estudio de secuenciación exómica en trío, resultado requerido para recepción del paciente en centro de trasplante de células hematopoyéticas.

El objetivo inicial del manejo consistió en optimizar el estado nutricional. Se ajustaron los parámetros en la nutrición parenteral para paciente con falla intestinal y se inició fórmula de aminoácidos libres a bajo volumen, logrando ganancia de peso paulatina, pese al alto gasto fecal. Se adicionó terapia farmacológica de primera línea con metilprednisolona, sin embargo, luego de cuatro semanas de tratamiento el paciente persistió con alto gasto fecal. Se añadió ciclosporina presentando hepatotoxicidad y persistencia de diarrea requiriendo cambio de manejo por tacrolimus, ante no disponibilidad de sirolimus.

A los 7 meses de vida, recibiendo manejo inmunosupresor y soporte nutricional mixto, presentó trombosis extensa con afectación de múltiples vasos, haciendo imposible la consecución de un nuevo acceso venoso, se suspendió nutrición parenteral lo que llevó a la muerte del paciente.

Posterior al fallecimiento, se recibió el reporte de la secuenciación con hallazgo de una variante en estado hemicigoto en el gen FOXP3: c.2T>C (p.Met1Thr), identificada en el paciente y su madre. Dicha variante no había sido reportada previamente en la literatura ni en las bases de datos consultadas.

\section{Discusión}

El síndrome IPEX es causado por mutaciones del gen FOXP3. La pérdida de función en FOXP3 conlleva a desregulación inmunitaria, caracterizada por respuesta exagerada a estímulos, mediante la activación de receptores de antígenos de los linfocitos T (TCR), liberación de mediadores inflamatorios, ocasionando daño tisular y producción de auto-anticuerpos ${ }^{3,7}$.

Los datos en cuanto a la prevalencia de esta entidad no son exactos, se estima que afecta a 1 de cada 1.600.000 personas a nivel mundial ${ }^{8}$, sin embargo, en Latinoamérica no se dispone de datos epidemiológicos.

La triada clásica de esta entidad consiste en enteropatía, diabetes mellitus tipo I y alteraciones derma- 
Tabla 2. Causas de diarrea crónica en el primer año de vida

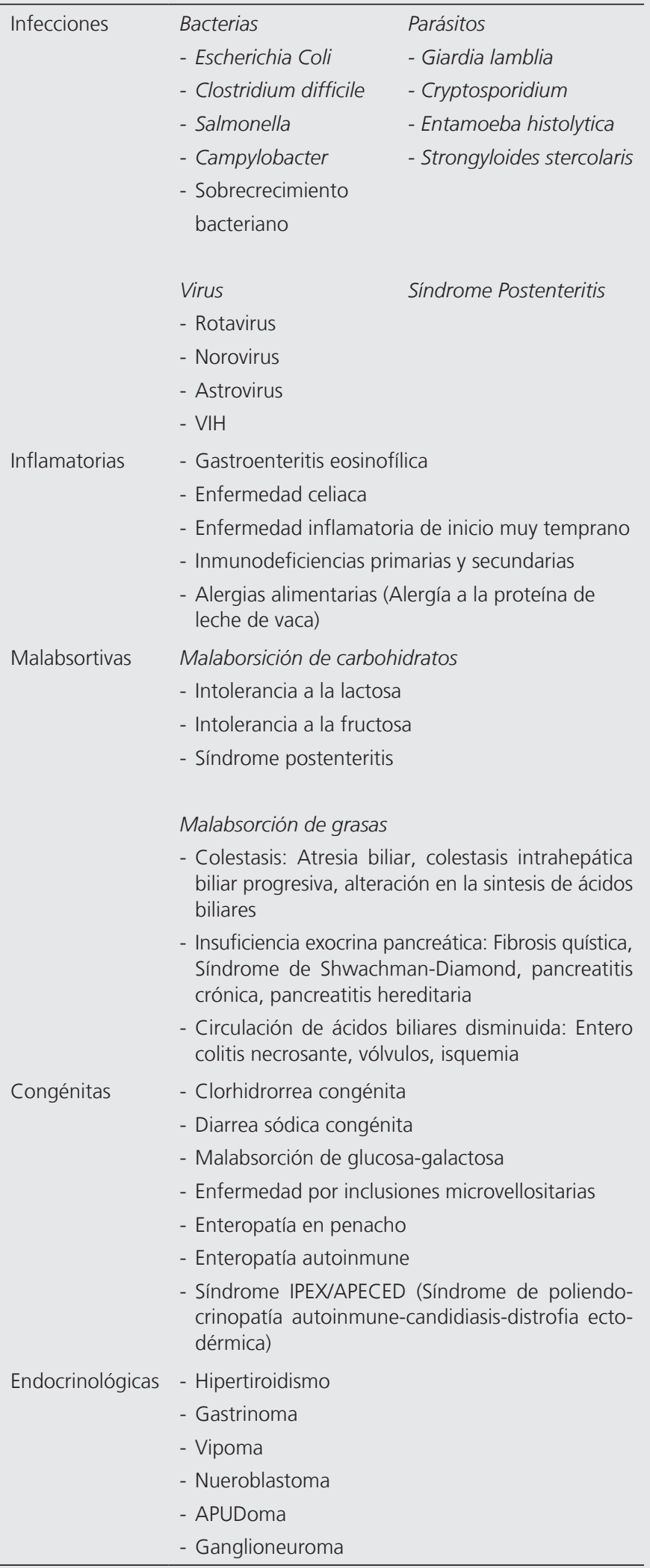

Adaptado de Rishi Gupta. Diarrhea. En Wyllie, Robert, MD; Hyams, Jeffrey S., MD; Kay, Marsha, MD. Pediatric Gastrointestinal and Liver Disease. 5ta edición. Elsevier, 2016. p. 104-14. tológicas (eczema difuso y/o alopecia). Se han descrito otras manifestaciones de carácter autoinmune como hipotiroidismo, hepatitis, alteraciones hematológicas (anemia, trombocitopenia y/o neutropenia) y alteraciones renales ${ }^{3}$. Sin embargo, las alteraciones endocrinológicas y en piel pueden ser tardías y no son indispensables para realizar el diagnóstico, como en nuestro paciente.

La enteropatía es característica en todos los casos, por lo que el primer síntoma suele ser diarrea. Su relevancia recae en la severidad de esta, que conlleva a falla intestinal y desnutrición proteico-calórica severa. La diarrea es de inicio temprano, antes de los 3 meses de vida, de duración mayor a 6 semanas, acuosa y puede estar acompañada de esteatorrea. No es usual la presencia concomitante de deposiciones con sangre9.

El diagnóstico de esta entidad radica en la sospecha clínica ante un varón con los hallazgos clínicos descritos y la identificación de una mutación en FOXP3. Es primordial un abordaje clínico adecuado, donde se investiguen los antecedentes familiares del paciente. En los exámenes diagnósticos, inespecíficos, el recuento linfocitario suele ser normal en contraste con elevación de los eosinófilos en sangre periférica. Con relación a la inmunidad celular, se puede evidenciar elevación discreta del conteo de $\mathrm{CD}^{+}$y a nivel humoral los títulos de IgG, IgM e IgA son normales, a excepción de los niveles de $\operatorname{IgE}$ que frecuentemente se encuentran altamente elevados. Anticuerpos anti-enterocito y anti-colonocito tipo IgG pueden estar presentes ${ }^{3}$. Suelen sobre diagnosticarse patologías mas frecuentes como la alergia a proteína de leche de vaca, retardando el diagnóstico. Otras enfermedades que deben considerarse en el diagnóstico diferencial de la diarrea crónica de inicio temprano son las infecciosas y la fibrosis quística entre otras (tabla 2$)^{10}$.

Ante la sospecha de enteropatía autoinmune es mandatorio la realización de endoscopia y colonoscopia con toma de biopsias. Duclaux-Loras y cols, realizaron un estudio multicéntrico retrospectivo con 30 pacientes con Síndrome IPEX. De estos, 19 fueron llevados a endoscopia diagnóstica, encontrándose inflamación severa del tracto gastrointestinal superior y del colon ${ }^{11}$. Los hallazgos histológicos clásicos son: atrofia severa de vellosidades, hiperplasia de la lámina propia con infiltrado inflamatorio mononuclear e hiperplasia de las criptas con evidencia de cuerpos apoptóticos, como se evidenció en el paciente. Estos hallazgos no son patognomónicos de IPEX y pueden encontrarse en enteropatía autoinmune de diferente etiología ${ }^{12}$.

La variante en el gen FOXP3 reportada en nuestro paciente es de frecuencia poblacional desconocida. Según los criterios del Colegio Americano de Genética Médica se clasifica como probablemente patogénica. Este cambio corresponde a una variante frameshift que 
altera el primer aminoácido de la proteína en una posición altamente conservada. Dicha variante fue analizada con 13 predictores bioinformáticos (Polyphen, mutation tester, SIFT, entre otros), de los cuales 12 la clasifican como patogénica. El análisis bioinformático junto con el reporte de la literatura de otras variantes en la misma posición, indican que provoca una pérdida del primer aminoácido metionina, por un desplazamiento del marco de lectura, causando un cambio en la secuencia de la proteína con pérdida de varios dominios funcionales de la misma.

Este caso ilustra la importancia del estudio genético en patologías de muy baja prevalencia, donde el diagnóstico etiológico es complejo por la diversidad y sobreposición de síntomas que comparte con otras entidades clínicas. La secuenciación exómica completa es una herramienta fundamental en enfermedades monogénicas, donde no es posible el diagnóstico mediante otras pruebas. Su importancia radica en establecer pronóstico, tratamiento y en ofrecer asesoramiento genético a la familia, con el objetivo de brindar riesgos reproductivos y opciones disponibles para disminuir repetición en la descendencia. De esta manera se disminuyen costos a futuro y el impacto emocional a nivel familiar y social de otro hijo con la enfermedad.

El abordaje del paciente requiere un equipo multidisciplinario, instaurándose oportunamente medidas de soporte, con el fin de corregir las alteraciones metabólicas y electrolíticas secundarias. Se plantea como primera línea de manejo la administración de glucocorticoides para disminuir la respuesta inflamatoria, aunque estudios recientes sugieren el uso de Sirolimus (rapamicina) como primera línea con resultados prometedores ${ }^{13}$. En nuestro país no se dispone aún de este medicamento.

Además del uso de esteroides, se han evaluado otras terapias inmunomuladoras como Ciclosporina, Tacrolimus y Ciclofosfamida; y con menos frecuencia el uso de Azatioprina y Micofenolato Mofetil. La administración conjunta de estos medicamentos ha demostrado eficacia como terapia de mantenimiento y disminuye a largo plazo las complicaciones derivadas del uso crónico del esteroide? .

Los pacientes con refractariedad al manejo inmunosupresor son candidatos a terapia biológica con Infliximab, Adalimumab y Abatacept. Hasta la fecha el trasplante de médula ósea ha demostrado ser la única terapia curativa en pacientes con síndrome IPEX 9

La tasa de sobrevida a 15 años en pacientes sometidos a trasplante de médula ósea fue de $73,2 \%$, en comparación a $65,1 \%$ en aquellos que sólo recibieron manejo inmunosupresor, por tanto hasta el momento constituye la mejor opción terapéutica en pacientes con esta enfermedad ${ }^{14}$.

\section{Conclusiones}

La diarrea crónica de inicio temprano, en contexto de Síndrome IPEX, constituye un caso de interés por tratarse de una enfermedad infrecuente. La sospecha clínica puede generarse a partir de una buena historia clínica, la interpretación de la historia familiar y los hallazgos de laboratorio y anatomopatológicos. Es imperativo confirmar el diagnóstico a través del estudio molecular, que permite describir mutaciones conocidas y nuevas, como en nuestro caso. Esto logrará caracterizar mejor la enfermedad para oportunamente brindar manejo integral y asesoramiento genético a las familias.

\section{Responsabilidades Éticas}

Protección de personas y animales: Los autores declaran que los procedimientos seguidos se conformaron a las normas éticas del comité de experimentación humana responsable y de acuerdo con la Asociación Médica Mundial y la Declaración de Helsinki.

Confidencialidad de los datos: Los autores declaran que han seguido los protocolos de su centro de trabajo sobre la publicación de datos de pacientes.

Derecho a la privacidad y consentimiento informado: Los autores han obtenido el consentimiento informado de los pacientes y/o sujetos referidos en el artículo. Este documento obra en poder del autor de correspondencia.

\section{Conflicto de intereses}

Los autores declaran no tener conflicto de intereses. 


\section{Referencias}

1. d'Hennezel E, Dhuban KB, Torgerson T, Piccirillo C. The immunogenetics of immune dysregulation, polyendocrinopathy, enteropathy, $\mathrm{X}$ linked (IPEX) syndrome. J Med Genet. 2012;49(5):291-302.

2. González Parias JL, Duque Giraldo VE, Velásquez-Lopera MM. FOXP3: Controlador maestro de la generación y función de las células reguladoras naturales. Inmunología 2010;29(2): 74-84.

3. Walker WA, Kleinman RE. Walker's Pediatric Gastrointestinal Disease: Physiology, Diagnosis, Management. Vol. 5th Edition. Hamilton, Ont: PMPH USA, Ltd; 2008.

4. Blanco Quirós A, Arranz Sanz E, Bernardo Ordiz D, Garrote Adrados JA. From autoimmune enteropathy to the IPEX (immune dysfunction, polyendocrinopathy, enteropathy, $\mathrm{X}$-linked) syndrome. Allergol Immunopathol (Madr). 2009;37(4):
208-15.

5. OMIM - Online Mendelian Inheritance in Man [Internet]. [citado 9 de enero de 2020]. Disponible en: https://omim.org/

6. Hughes H, Kahl L. Manual Harriet Lane de pediatría. 21. a ed. Elsevier; 2018. 1244 p.

7. Pesenacker AM, Cook L, Levings MK. The role of FOXP3 in autoimmunity. Curr Opin Immunol. 2016;43:16-23.

8. Reference GH. IPEX syndrome [Internet]. Genetics Home Reference. [citado el 9 de enero de 2020]. Disponible en: https:// ghr.nlm.nih.gov/condition/immunedysregulation-polyendocrinopathyenteropathy-x-linked-syndrome

9. Ahmed Z, Imdad A, Connelly JA, Acra S. Autoimmune Enteropathy: An Updated Review with Special Focus on Stem Cell Transplant Therapy. Dig Dis Sci. 2019;64(3):643-54.

10. Rishi Gupta. Diarrhea. En Wyllie, Robert, MD; Hyams, Jeffrey S., MD; Kay, Marsha, MD. Pediatric Gastrointestinal and Liver Disease. 5ta edición. Elsevier, 2016. p. 104-14.
11. Duclaux-Loras R, Charbit-Henrion F, Neven B, Nowak J, CollardeauFrachon S, Malcus C, et al. Clinical Heterogeneity of Immune Dysregulation, Polyendocrinopathy, Enteropathy, $\mathrm{X}$-Linked Syndrome: A French Multicenter Retrospective Study. Clin Transl Gastroenterol. 2018;9(10):e201.

12. Masia R, Peyton S, Lauwers GY, Brown I. Gastrointestinal Biopsy Findings of Autoimmune Enteropathy: A Review of 25 Cases. Am J Surg Pathol. 2014;38(10):1319.

13. Bacchetta R, Barzaghi F, Roncarolo M-G. From IPEX syndrome to FOXP3 mutation: a lesson on immune dysregulation. Ann N Y Acad Sci. 2018;1417(1):5-22.

14. Barzaghi F, Amaya Hernandez LC, Neven B, et al. Long-term follow-up of IPEX syndrome patients after different therapeutic strategies: An international multicenter retrospective study. J Allergy Clin Immunol. 2018;141(3):1036-1049.e5. 